\title{
Atividade física e fatores associados em técnico- administrativos de uma universidade pública do sul do Brasil
}

\author{
Physical activity and associated factors in public university employees of \\ southern Brazil
}

\section{AUTORES \\ Tiago Silva dos Santos 1 (iD \\ Samuel Völz Lopes ${ }^{1}$ if \\ Eduardo Lucia Caputo ${ }^{1}$ iD \\ Jeferson Santos Jerônimo ${ }^{1}$ (iD \\ Marcelo Cozzensa da Silva ${ }^{1}$ (iD \\ 1 Universidade Federal de Pelotas, Escola Superior de Educação Física, Pelotas, Rio Grande do Sul, Brasil.}

\section{CONTATO}

Tiago Silva dos Santos

ss.tiago.s@gmail.com

Escola Superior de Educação Física, Universidade Federal de Pelotas.

Rua Luiz de Camões, 625, Pelotas, Rio

Grande do Sul, Brasil. CEP: 96055-630.

DOI

$10.12820 /$ rbafs. $23 \mathrm{e} 0028$

\begin{abstract}
RESUMO
O objetivo do estudo foi identificar a prevalência de atividade física (AF) e fatores associados em trabalhadores técnico-administrativos (TA) de uma universidade pública do sul do Brasil. Realizou-se um estudo transversal com TA, selecionado em múltiplos estágios. Foi utilizado um questionário estruturado autopreenchível com variáveis sociodemográficas, nutricional, comportamentais e de trabalho. O IPAQ versão longa foi utilizado para medir a AF, nos domínios de lazer, deslocamento e AF total. A regressão logística multinomial com modelo hierárquico foi utilizada para testar as associações, adotou-se valor de $\mathrm{p}<0,05$. As prevalências de $\mathrm{AF}$ total, no lazer e no deslocamento foram de $61,1 \%, 56,8 \%$ e $26,2 \%$, respectivamente. Homens foram mais ativos que as mulheres no lazer ( $p$ $=0,047)$, no deslocamento $(\mathrm{p}=0,033)$ e na AF total $(\mathrm{p}=0,015)$. Idade mais avançada foi associada a maior $\mathrm{AF}$ no deslocamento $(\mathrm{p}=0,003)$. Maior escolaridade foi associada com maior prática de $\mathrm{AF}$ total $(p=0,013)$ e de lazer $(p=0,014)$. Situação conjugal solteiro demonstrou associação somente com o domínio do deslocamento $(\mathrm{p}=0,014)$. Os adultos eutróficos apresentaram maior prática de AF no lazer $(p=0,027)$. No mesmo sentido, os TA que afirmaram não fumar estiveram associados com AF no lazer $(p=0,002)$. Foi possível concluir que TA apresentam comportamentos distintos de acordo com cada domínio de $\mathrm{AF}$, sendo necessárias ações pontuais que promovam a adoção do comportamento fisicamente ativo entre os funcionários da instituição.
\end{abstract}

Palavras-chave: Atividade física; Trabalhadores; Universidades.

\section{ABSTRACT}

The aim of the study was to identify the prevalence of physical activity $(P A)$ and associated factors in administrative workers $(A W)$ of a university in the south of Brazil. A cross-sectional study was carried out with a sample of university workers, using a multi-stage sampling process. Data were collected through a structured auto filled questionnaire containing sociodemographic, nutritional, behavioral and work variables. For the measurement of the outcome variable, level of $P A$, the IPAQ long version was used. Data regarding the leisure-time, displacement and total PA domains were used. To verify the association between the outcome variable and independent variables, a multinomial logistic regression was used, and a hierarchical analysis model was used. A p value $<0.05$ was stipulated to assume association between the variables. The prevalence of $P A$ for each domain was, respectively, $61.1 \%$ (total), $56.8 \%$ (leisure-time) $26.2 \%$ (transport). Men were more active than women in the domains of leisure-time $(p=0.047)$, transport $(p=0.033)$. Higher schooling was associated with greater practice of total $P A(p=0.013)$ and leisure-time $(p=0.014)$. Single marital status demonstrated association only with the trasnport domain $(p=0.014)$. Subjects classified in the category of eutrophic BMI presented greater leisure-time practice $(p=0.027)$. In the same sense, $A W$ who reported not smoking were associated with greater $P A$ in leisure-time $(p=0.002)$. It was possible to conclude that administrative workers present different behaviors according to each domain of $P A$, and specific actions are required to promote the adoption of physically active behavior among employees of the institution.

Keywords: Physical activity; Workers; Universities.

\section{Introdução}

A atividade física (AF) não é simplesmente uma questão de escolha individual, mas refere-se a um comportamento complexo e multidimensional que envolve o movimento humano, resultante da relação entre atributos fisiológicos, psicológicos, sociais e ambientais ${ }^{1}$. Desta forma, podendo ser representada e mensurada em diferentes domínios, como AF ocupacional, AF doméstica, AF de lazer, AF de deslocamento, possibilitando o estudo em diferentes populações, como a de trabalhadores ${ }^{1}$. 
A associação entre AF e doenças crônicas não transmissíveis (DCNT) é um dos principais alvos dos estudos da área de saúde nas últimas décadas, visto o risco que ser inativo acarreta a população ${ }^{2}$. A preocupação com os baixos níveis de AF relatados pela população tem levado várias agências de saúde ao redor do mundo a se dedicar a essa temática ${ }^{3}$. E neste sentido, estudos tem identificado uma estreita relação entre níveis aumentados de AF, melhor qualidade de vida e bem-estar da população em geral ${ }^{2,3}$. Entre estes ganhos em saúde, é possível destacar: aumento da capacidade cardiorrespiratório, melhora da composição corporal, saúde óssea, capacidade funcional e função cognitiva ${ }^{2}$.

Em relação à classe trabalhadora, compreender o cotidiano desta população se faz necessário, visto que a rotina laboral pode influenciar na qualidade de vida destes indivíduos. E por muitas vezes aliado a uma alta carga de trabalho, é possível observar condições precárias de estrutura socioeconômica e familiar, o que acaba por afastar o trabalhador de um estilo de vida ativo e saudável ${ }^{4}$.

Sabe-se ainda, que adultos em idade produtiva parecem estar expostos a barreiras e determinantes ainda desconhecidos, que dificultam a adoção de um estilo de vida ativo ${ }^{5}$. Neste sentido, Ogata et al. ${ }^{6}$ salienta a importância a ser despendida à classe trabalhadora ao identificar um modelo atual de trabalho que sacrifica a saúde em busca do sucesso profissional.

Todavia, Nahas et al. ${ }^{7}$ e Silva et al. ${ }^{8}$ enaltecem a importância que a adoção de hábitos de vida saudáveis, como a prática regular de AF, pode auxiliar a minimizar os danos à saúde gerados pelas intensas e longas jornadas de trabalho, e assim atuar na melhoria do desempenho das atividades laborais. Nahas et al. ${ }^{7}$ afirma ainda, que a manutenção de um estilo de vida ativo auxilia o trabalhador a adoecer com menor frequência e por consequência gera uma maior produtividade.

No Brasil, o sistema de Vigilância de Fatores de Risco e Proteção para Doenças Crônicas por Inquérito Telefônico (VIGITEL) ao analisar dados referentes ao conjunto das capitais do país em 2015, identificou uma variação na frequência de adultos que praticam AF no lazer equivalente a pelo menos 150 minutos de AF moderada por semana entre 30,7\% em São Paulo e 52,1\% no Distrito Federal ${ }^{9}$. Tais resultados são similares àqueles encontrados em pesquisas que buscaram avaliar a prática de AF no lazer em população de trabalhadores, como é o caso de estudos realizados no estado de Santa Catarina $\left(48,3 \%{ }^{10} ; 30,8 \%^{11}\right)$ e no estado de São Paulo $\left(41 \%{ }^{4}\right)$. Assim, o presente estudo teve por objetivo identificar a prevalência de AF e fatores associados em trabalhadores técnico-administrativos de uma universidade pública do sul do Brasil.

\section{Métodos}

O presente estudo foi redigido seguindo os pressupostos do checklist $\mathrm{STROBE}^{12}$. Foi realizado um estudo de delineamento transversal com uma amostra composta por servidores técnico-administrativos da Universidade Federal de Pelotas (UFPEL) entre 2014 e 2015. Dentre as características do local do estudo, é importante salientar que a UFPel está situada, em diversos campi, alocados nos municípios de Pelotas e Capão do Leão, ambos distantes a, aproximadamente 250 quilômetros ao sul de Porto Alegre, capital do estado do Rio Grande do Sul.

Após a autorização da Pró-Reitoria de Gestão de Pessoas da universidade obteve-se a listagem oficial dos técnico-administrativos, e então, ocorreu a listagem e verificação de todas as unidades operacionais da universidade. $\mathrm{Na}$ sequência, foi realizado um processo de amostragem estratificado (reitoria; cursos das áreas da saúde; ciências humanas; sociais; exatas; da terra; biológicas; agrárias; engenharias; linguística; letras e; artes) e com probabilidade proporcional ao tamanho (número de funcionários) entre todas as unidades da UFPEL, em todos os turnos de trabalho. Desta forma, foi possível estimar uma população de 1.356 servidores técnico-administrativos.

O processo de amostragem realizou-se em múltiplos estágios. Primeiramente, todas as unidades acadêmicas e administrativas foram agrupadas por áreas afins, sendo elas: reitoria e unidades administrativas, ciências exatas, humanas e biológicas. As áreas foram então ordenadas, de maior a menor, de acordo com o número de técnico-administrativos que trabalhavam em cada uma delas.

Foi realizado um cálculo de tamanho amostral para estudo de prevalência e associação. $\mathrm{O}$ maior valor encontrado foi de 369 pessoas (cálculo para prevalência: tamanho da população $=2.000$, prevalência de $55 \%$ de AF no lazer, erro aceitável de $5 \%$ e um nível de confiança de $95 \%$, acrescidos de $10 \%$ para controle de perdas e $5 \%$ para recusas).

Posteriormente ao cálculo de tamanho amostral, foi realizado um sorteio para cada unidade da universidade a partir da lista dos técnico-administrativos em ordem alfabética, desta forma procurou-se garantir uma total representatividade da população a ser estudada. 
A partir da obtenção do número de sujeitos a serem amostrados foi estabelecido um pulo sistemático de três indivíduos, a começar por um indivíduo sorteado da maior área de agrupamento até atingir-se o número de amostra estimado. Todos os indivíduos sorteados para participar da coleta foram procurados em suas unidades de trabalho e informados dos objetivos, riscos e contribuições do estudo e, então, convidados a participar mediante a apresentação e solicitação da assinatura do Termo de Consentimento Livre e Esclarecido (TCLE), para posterior preenchimento do questionário. Quanto àqueles participantes não encontrados inicialmente, foram realizadas, pelo menos, outras três tentativas de contato no local de trabalho, e em caso de falha, buscou-se ainda contato telefônico ou via e-mail para viabilizar a coleta de dados.

Foram excluídos da amostra aqueles indivíduos que se apresentavam afastados do trabalho por motivos de doença mental que os incapacitasse de responder o instrumento de pesquisa, aqueles que estivessem em internação hospitalar ou ainda aqueles técnico-administrativos que no período de coleta de dados se encontrassem em meio a realização de cursos de capacitação (especialização, mestrado, doutorado) fora do município de Pelotas.

A coleta de dados ocorreu no período de julho de 2014 a janeiro de 2015 e se deu através de questionário estruturado auto preenchível contendo variáveis sociodemográficas - sexo (masculino, feminino), idade (anos completos), cor da pele (relatada pelo entrevistado), situação conjugal (casado/vive com companheiro, solteiro, separado, viúvo) e escolaridade (anos de estudo), nutricional - índice de massa corporal [IMC], calculado pelo peso $[\mathrm{kg}]$ referido, dividido pela altura $[\mathrm{cm}]$ referida elevada ao quadrado (eutrófico, sobrepeso e obesidade $)^{13}$, comportamentais - uso de medicamentos (sim, não), tabagismo (sim, não) e alcoolismo (escore obtido através do CAGE Questionnaire) ${ }^{14}$, e de trabalho - carga horaria semanal de trabalho (até 40 horas semanais, + de 40 horas semanais) e acidentes de trabalho (sim, não).

Para mensuração da principal variável de interesse, o nível de AF, foi utilizado o International Physical Activity Questionnaire (IPAQ) versão longa, validado no Brasil por Matsudo et al. ${ }^{15}$. Foram analisados os dados das seções de lazer e deslocamento, onde ocorreu o cálculo do tempo de AF total (lazer + deslocamento) semanal dispendidos em atividades moderadas e vigorosas, bem como cálculos independentes para níveis de AF nos domínios de lazer e deslocamento. Sendo que, para atividades vigorosas foi realizada a multiplicação por dois do tempo relatado. Já o ponto de corte utilizado para categorizar ativos e não ativos foi de 150 minutos semanais de $\mathrm{AF}^{16}$.

Para estruturação do banco de dados foi utilizado o programa EpiData 3.1, onde foi realizada a dupla digitação e verificação de inconsistências entre os bancos. Enquanto a análise estatística foi realizada através do pacote Stata 13.0 com processo dividido em três etapas. Primeiramente ocorreu a análise descritiva das variáveis (cálculo de médias e desvio padrão para as variáveis contínuas e de proporções e Intervalos de Confiança para as categóricas). Em um segundo momento, foram conduzidas análises bivariadas entre as variáveis independentes e o nível de AF total, no lazer e no deslocamento, separadamente para cada domínio, através do teste de Qui-Quadrado de Pearson para variáveis categóricas nominais e teste de Tendência linear para variáveis categóricas ordinais, sendo considerado um valor $\mathrm{p}<0,05$ para associação. Na última parte da análise foi realizada uma regressão logística multinomial, respeitando a hierarquia de determinação do desfecho a ser estudado.

O modelo hierárquico de análise foi composto por três níveis: o primeiro constituído pelas variáveis sexo, idade, cor da pele, situação conjugal e escolaridade; o segundo contendo as variáveis IMC, tabagismo, alcoolismo e uso de medicamento; o terceiro nível da cadeia composto por carga horária semanal de trabalho, acidente de trabalho e tempo como técnico-administrativos.

Cada um dos desfechos (AF lazer, AF deslocamento e AF total) foi avaliado por meio de regressão logística, sendo mantido no modelo variáveis com valor $p$ $\leq 0,2$ para controle de confusão. Foram consideradas associadas ao desfecho aquelas variáveis que apresentaram valor $\mathrm{p}<0,05$.

O projeto de pesquisa foi aprovado pelo Comitê de Ética e Pesquisa (CEP) da Escola Superior de Educação Física da UFPel, sob o parecer número 725.405. O estudo também está de acordo com a Resolução 196/96 do Conselho Nacional de Saúde, cumprindo todos os deveres éticos que cabe a comunidade cientifica e assegurando todos e quaisquer direitos cabíveis aos sujeitos da pesquisa.

\section{Resultados}

De um total de 459 técnico-administrativos sorteados, 371 consentiram em participar do estudo e responderam o questionário. Foram consideradas perdas e recusas um total de $19,2 \%$, sendo de características simila- 
res entre sexo e idade dos amostrados.

A tabela 1 apresenta as características sociodemográficas, comportamentais e de trabalho dos participantes. A maior parte da amostra era do sexo feminino $(57,4 \%)$, de cor de pele branca $(83,6 \%)$, casado $(66,1 \%)$, em estado nutricional equivalente a categoria de sobrepeso $(41,4 \%)$ e pertencia a faixa etária 50-59 anos (31,0\%). Com relação à prática de $\mathrm{AF}$ total, $61,1 \%$ atingiram as recomendações mínimas de atividade física para benefícios à saúde, $56,8 \%$ foram considerados suficientemente ativos no lazer e $26,2 \%$ no deslocamento. Com relação às características de trabalho, $53,1 \%$ da amostra tinha até 14 anos de trabalho, 50,3\% trabalhava até 40 horas por semana e $84,5 \%$ nunca teve acidentes no trabalho.

A Tabela 2 apresenta o resultado da análise bruta realizada entre variáveis independentes e $\mathrm{AF}$ total e nos domínios de lazer e deslocamento. A AF total esteve associada com as variáveis sexo $(p=0,014)$ e escolaridade $(\mathrm{p}=0,014)$. No domínio lazer, $\mathrm{AF}$ foi associada com sexo $(p=0,023)$, idade $(p=0,038)$, escolaridade $(\mathrm{p}=0,013)$ e fumo $(\mathrm{p}=0,005)$, e no domínio do deslocamento, com idade $(p=0,007)$, fumo $(p=0,009)$ e alcoolismo $(p=0,008)$.

O resultado da análise ajustada se encontra na $\mathrm{Ta}$ bela 3. Com relação a AF total, as associações encontradas na análise bruta com sexo $(p=0,015)$ e escolaridade $(\mathrm{p}=0,013)$ foram mantidas. Indivíduos do sexo masculino tiveram $88,0 \%$ mais chance de serem ativos no lazer que seus pares e à medida que aumentou a escolaridade, maior a prevalência de prática. No domínio do lazer, sexo $(\mathrm{p}=0,047)$, escolaridade ( $\mathrm{p}=$ $0,014)$ e fumo $(p=0,002)$, mantiveram-se associadas ao desfecho apresentando o mesmo padrão encontrado à associação com AF total. Trabalhadores não fumantes tiveram $342 \%$ mais chance de praticar AF no lazer que seus pares fumantes. Além disso, a medida que os indivíduos trocaram de categoria de IMC (de obesidade - categoria de base - à eutrofia) mais ativos no lazer se mostraram $(p=0,027)$. Em relação ao domínio do deslocamento, a variável idade $(\mathrm{p}=0,003)$, manteve a associação anteriormente encontrada na análise bruta. Ademais, maior prevalência de AF nesse domínio foi encontrada entre técnico-administrativos do sexo masculino $(p=0,033)$ e solteiros $(p=0,014)$.

\section{Discussão}

O presente estudo se propôs a verificar a prevalência e os fatores associados à prática de $\mathrm{AF}$ em servidores
Tabela 1 - Características sociodemográficas, comportamentais e de trabalho de servidores técnico-administrativos de uma universidade federal do sul do Brasil ( $\mathrm{n}=371)$.

\begin{tabular}{|c|c|}
\hline Variáveis & n (\%) \\
\hline \multicolumn{2}{|l|}{ Atividade física total } \\
\hline$\geq 150 \mathrm{~min} . / \mathrm{semana}$ & $187(61,1)$ \\
\hline$<149 \mathrm{~min} . / \mathrm{semana}$ & $119(38,9)$ \\
\hline \multicolumn{2}{|l|}{ Atividade física no lazer } \\
\hline$\geq 150 \mathrm{~min} . / \mathrm{semana}$ & $137(56,8)$ \\
\hline$<149 \mathrm{~min} . / \mathrm{semana}$ & $180(43,2)$ \\
\hline \multicolumn{2}{|c|}{ Atividade física no deslocamento } \\
\hline$\geq 150 \mathrm{~min} . / \mathrm{semana}$ & $89(26,2)$ \\
\hline$<149 \mathrm{~min} . / \mathrm{semana}$ & $251(73,8)$ \\
\hline \multicolumn{2}{|l|}{ Sexo } \\
\hline Feminino & $213(57,4)$ \\
\hline Masculino & $158(42,6)$ \\
\hline \multicolumn{2}{|l|}{ Idade (anos) } \\
\hline $20-29$ & $42(11,3)$ \\
\hline $30-39$ & $88(23,7)$ \\
\hline $40-49$ & $85(22,9)$ \\
\hline $50-59$ & $115(31,0)$ \\
\hline 60 ou mais & $41(11,1)$ \\
\hline \multicolumn{2}{|l|}{ Cor da pele } \\
\hline Não-branca & $61(16,4)$ \\
\hline Branca & $310(83,6)$ \\
\hline \multicolumn{2}{|c|}{ Escolaridade (anos de estudo) } \\
\hline $0-12$ & $52(15,3)$ \\
\hline $13-17$ & $119(34,9)$ \\
\hline$\geq 18$ & $170(49,8)$ \\
\hline \multicolumn{2}{|l|}{ Situação conjugal } \\
\hline Casado & $245(66,1)$ \\
\hline Solteiro & $126(33,9)$ \\
\hline \multicolumn{2}{|c|}{ Índice de massa corporal } \\
\hline Obesidade & $81(22,4)$ \\
\hline Sobrepeso & $150(41,4)$ \\
\hline Eutrófico & $131(36,2)$ \\
\hline \multicolumn{2}{|l|}{ Fumo } \\
\hline Sim & $50(13,5)$ \\
\hline Não & $320(86,5)$ \\
\hline \multicolumn{2}{|l|}{ Álcool } \\
\hline Não & $326(95,6)$ \\
\hline $\operatorname{Sim}$ & $15(4,4)$ \\
\hline \multicolumn{2}{|l|}{ Uso de medicamento } \\
\hline Não & $308(83,5)$ \\
\hline $\operatorname{Sim}$ & $61(16,5)$ \\
\hline \multicolumn{2}{|l|}{ Anos de trabalho } \\
\hline Até 14 & $197(53,1)$ \\
\hline$\geq 15$ & $174(46,9)$ \\
\hline \multicolumn{2}{|c|}{ Carga horária semanal (horas) } \\
\hline Até 40 & $185(50,3)$ \\
\hline 41 ou mais & $183(49,7)$ \\
\hline \multicolumn{2}{|l|}{ Acidente de trabalho } \\
\hline Não & $311(84,5)$ \\
\hline $\operatorname{Sim}$ & $57(15,5)$ \\
\hline
\end{tabular}


Tabela 2 - Análise bruta entre AF total, lazer e deslocamento e variáveis independentes de servidores técnico-administrativos de uma universidade federal do sul do Brasil $(\mathrm{n}=371)$.

\begin{tabular}{|c|c|c|c|c|c|c|}
\hline \multirow[t]{2}{*}{ Variáveis } & \multicolumn{2}{|l|}{$\begin{array}{l}\text { AF Total } \\
(\mathrm{n}=306)\end{array}$} & \multicolumn{2}{|l|}{$\begin{array}{l}\text { AF Lazer } \\
(\mathrm{n}=317)\end{array}$} & \multicolumn{2}{|l|}{$\begin{array}{l}\text { AF Deslocamento } \\
(\mathrm{n}=340)\end{array}$} \\
\hline & OR (IC95\%) & $\mathrm{p}$ & OR (IC95\%) & $\mathrm{p}$ & OR (IC95\%) & $\mathrm{p}$ \\
\hline \multicolumn{7}{|l|}{ Sexo } \\
\hline Feminino & 1 & & 1 & & 1 & \\
\hline Masculino & $1,81(1,13-2,91)$ & $0,014^{\mathrm{a}}$ & $1,68(1,07-2,63)$ & $0,021^{\mathrm{a}}$ & $1,47(0,90-2,40)$ & 0,115 \\
\hline \multicolumn{7}{|l|}{ Idade (anos) } \\
\hline $20-29$ & 1 & & 1 & & 1 & \\
\hline $30-39$ & $0,43(0,18-1,04)$ & 0,061 & $0,37(0,17-0,84)$ & $0,017^{\mathrm{a}}$ & $1,01(0,37-2,71)$ & 0,987 \\
\hline $40-49$ & $0,47(0,19-1,15)$ & 0,101 & $0,32(0,14-0,73)$ & $0,007^{\mathrm{a}}$ & $1,67(0,64-4,34)$ & 0,288 \\
\hline $50-59$ & $0,48(0,20-1,15)$ & 0,102 & $0,35(0,16-0,78)$ & $0,011^{\mathrm{a}}$ & $2,15(0,86-5,38)$ & 0,101 \\
\hline 60 ou mais & $0,45(0,15-1,30)$ & 0,143 & $0,30(0,11-0,81)$ & $0,019^{a}$ & $2,61(0,88-7,71)$ & 0,082 \\
\hline \multicolumn{7}{|l|}{ Cor da pele } \\
\hline Não branca & 1 & & 1 & & 1 & \\
\hline Branca & $1,48(0,80-2,74)$ & 0,207 & $0,77(0,41-1,43)$ & 0,418 & $1,01(0,52-1,92)$ & 0,971 \\
\hline \multicolumn{7}{|c|}{ Escolaridade (anos de estudo) } \\
\hline $0-12$ & 1 & & 1 & & 1 & \\
\hline $13-17$ & $1,40(0,67-2,93)$ & 0,365 & $2,49(1,13-5,47)$ & $0,023^{a}$ & $0,72(0,33-1,56)$ & 0,413 \\
\hline$\geq 18$ & $2,24(1,10-4,57)$ & $0,026^{a}$ & $2,83(1,32-6,03)$ & $0,007^{a}$ & $0,57(0,27-1,19)$ & 0,139 \\
\hline \multicolumn{7}{|c|}{ Situação conjugal } \\
\hline Casado & 1 & & 1 & & 1 & \\
\hline Solteiro & $1,30(0,79-2,14)$ & 0,283 & $1,09(0,67-1,75)$ & 0,719 & $1,56(0,95-2,56)$ & 0,076 \\
\hline \multicolumn{7}{|l|}{ IMC } \\
\hline Obesidade & 1 & & 1 & & 1 & \\
\hline Sobrepeso & $2,44(1,31-4,52)$ & $0,005^{a}$ & $2,03(1,09-3,78)$ & $0,025^{\mathrm{a}}$ & $0,86(0,43-1,70)$ & 0,672 \\
\hline Eutrófico & $1,68(0,90-3,10)$ & 0,098 & $1,94(1,03-3,66)$ & $0,038^{\mathrm{a}}$ & $1,29(0,67-2,46)$ & 0,440 \\
\hline \multicolumn{7}{|l|}{ Fumo } \\
\hline Sim & 1 & & 1 & & 1 & \\
\hline Não & $1,8(0,88-3,65)$ & 0,105 & $3,24(1,43-7,33)$ & $0,005^{\mathrm{a}}$ & $0,41(0,21-0,81)$ & $0,016^{a}$ \\
\hline \multicolumn{7}{|l|}{ Álcool } \\
\hline Não & 1 & & 1 & & 1 & \\
\hline Sim & $1,89(0,50-7,15)$ & 0,349 & $1,12(0,36-3,43)$ & 0,835 & $4,00(1,34-11,9)$ & $0,018^{\mathrm{a}}$ \\
\hline \multicolumn{7}{|c|}{ Uso de medicamento } \\
\hline Não & 1 & & 1 & & 1 & \\
\hline Sim & $1,06(0,56-2,00)$ & 0,850 & $0,78(0,42-1,44)$ & 0,439 & $1,80(0,97-3,33)$ & 0,057 \\
\hline \multicolumn{7}{|c|}{ Anos de trabalho } \\
\hline Até 14 & 1 & & 1 & & 1 & \\
\hline$\geq 15$ & $0,78(0,49-1,25)$ & 0,317 & $0,72(0,46-1,14)$ & 0,161 & $1,33(0,82-2,16)$ & 0,248 \\
\hline \multicolumn{7}{|c|}{ Carga horária semanal (horas) } \\
\hline Até 40 & 1 & & 1 & & 1 & \\
\hline 41 ou mais & $1,23(0,77-1,95)$ & 0,372 & $0,92(0,59-1,45)$ & 0,746 & $0,96(0,59-1,56)$ & 0,883 \\
\hline \multicolumn{7}{|c|}{ Acidente de trabalho } \\
\hline Não & 1 & & 1 & & 1 & \\
\hline $\operatorname{Sim}$ & $1,62(0,83-3,19)$ & 0,156 & $1,15(0,62-2,13)$ & 0,654 & $1,71(0,92-3,19)$ & 0,085 \\
\hline
\end{tabular}

$\mathrm{a}=\mathrm{p}<0,005$.

técnico-administrativos de uma universidade federal do sul do Brasil. Os dados descritivos indicaram uma prevalência de $61,1 \%$ para AF total e de $56,8 \%$ para AF no lazer. É possível observar na literatura cientifica estudos similares como os realizados por Rocha et al. ${ }^{17}$ e Polisseni \& Ribeiro ${ }^{18}$, onde os autores encontraram respectivamente prevalências de $50,6 \%$ de AF total e $43,6 \%$ de AF no tempo de lazer em servidores de uni- 
Tabela 3 - Análise ajustada entre AF total, lazer e deslocamento e variáveis independentes de servidores técnico-administrativos de uma Universidade Federal do sul do Brasil ( $\mathrm{n}=371)$.

\begin{tabular}{|c|c|c|c|c|c|c|}
\hline \multirow[t]{2}{*}{ Variáveis } & \multicolumn{2}{|l|}{$\begin{array}{l}\text { AF Total } \\
(\mathrm{n}=306)\end{array}$} & \multicolumn{2}{|l|}{$\begin{array}{l}\text { AF Lazer } \\
(\mathrm{n}=317)\end{array}$} & \multicolumn{2}{|l|}{$\begin{array}{l}\text { AF Deslocamento } \\
(\mathrm{n}=340)\end{array}$} \\
\hline & OR (IC95\%) & $\mathrm{p}$ & OR (IC95\%) & $\mathrm{p}$ & OR (IC95\%) & $\mathrm{p}$ \\
\hline \multicolumn{7}{|l|}{ Sexo } \\
\hline Feminino & 1 & & 1 & & 1 & \\
\hline Masculino & $1,88(1,1-3,13)$ & $0,015^{\mathrm{a}}$ & $1,61(1,01-2,57)$ & $0,047^{\mathrm{a}}$ & $1,75(1,05-2,92)$ & $0,033^{a}$ \\
\hline \multicolumn{7}{|l|}{ Idade $(\operatorname{anos})^{*}$} \\
\hline $20-29$ & & & & & 1 & \\
\hline $30-39$ & & & & & $1,17(0,43-3,23)$ & 0,758 \\
\hline $40-49$ & & & & & $2,40(0,88-6,55)$ & 0,087 \\
\hline $50-59$ & & & & & $2,77(1,07-7,19)$ & $0,036^{\mathrm{a}}$ \\
\hline 60 ou mais & & & & & $3,25(1,06-9.90)$ & $0,039^{a}$ \\
\hline \multicolumn{7}{|l|}{ Escolaridade** } \\
\hline $0-12$ & 1 & & 1 & & & \\
\hline $13-17$ & $1,54(0,73-3,26)$ & 0,256 & $2,70(1,22-5,98)$ & $0,015^{a}$ & & \\
\hline$\geq 18$ & $2,36(1,15-4,86)$ & $0,020^{\mathrm{a}}$ & $2,98(1,39-6,38)$ & $0,005^{\mathrm{a}}$ & & \\
\hline \multicolumn{7}{|l|}{ Situação conjugal } \\
\hline Casado & 1 & & & & 1 & \\
\hline Solteiro & $1,49(0,87-2,55)$ & 0,147 & & & $1,94(1,14-3,29)$ & $0,014^{a}$ \\
\hline \multicolumn{7}{|c|}{ Índice de massa corporal } \\
\hline Obesidade & 1 & & 1 & & & \\
\hline Sobrepeso & $1,77(0,90-3,48)$ & 0,653 & $1,89(0,97-3,70)$ & 0,539 & & \\
\hline Eutrófico & $2,05(1,05-3,97)$ & 0,096 & $2,26(1,13-4,51)$ & $0,021^{\mathrm{a}}$ & & \\
\hline \multicolumn{7}{|l|}{ Fumo } \\
\hline Sim & 1 & & 1 & & 1 & \\
\hline Não & $2,02(0,91-4,50)$ & 0,084 & $4,42(1,69-11,54)$ & $0,002^{\mathrm{a}}$ & $1,82(0,88-3,76)$ & 0,105 \\
\hline \multicolumn{7}{|l|}{ Álcool } \\
\hline Não & & & & & 1 & \\
\hline Sim & & & & & $3,04(0,97-9,53)$ & 0,056 \\
\hline \multicolumn{7}{|l|}{ Anos de trabalho } \\
\hline Até 14 & & & & & 1 & \\
\hline$\geq 15$ & & & & & $0,61(0,29-1,28)$ & 0,188 \\
\hline \multicolumn{7}{|c|}{ Carga horária semanal (horas) } \\
\hline Até 40 & & & 1 & & & \\
\hline 41 ou mais & & & $0,68(0,4-1,13)$ & 0,139 & & \\
\hline \multicolumn{7}{|l|}{ Acidente de trabalho } \\
\hline Não & 1 & & 1 & & & \\
\hline Sim & $2,12(0,99-4,53)$ & 0,052 & $1,62(0,80-3,26)$ & 0,181 & & \\
\hline
\end{tabular}

*Teste de Tendência Linear;** Escolaridade em anos de estudo; $\mathrm{a}=\mathrm{p}<0,05$.

versidades da Bahia e Minas Gerais. Nahas ${ }^{19}$, ao avaliar industriários brasileiros, relatou que os estados do Norte e Sul do país apresentam frequências maiores de AF no lazer em relação a região Sudeste do país.

A associação entre gênero e AF demonstrou que indivíduos do sexo masculino apresentam comportamento mais ativo quando comparados às mulheres. Do ponto de vista geral, homens são mais ativos nos domínios lazer, trabalho e deslocamento, e as mulheres mais ativas no domínio doméstico ${ }^{20}$. Estudos realizados com servi- dores de uma universidade da Bahia ${ }^{17}$ e industriários do estado do Rio Grande do Sul ${ }^{21}$, corroboram esse achado.

A escolaridade foi associada a prática de AF tanto no ponto de vista total, quanto no de lazer, sendo verificada uma tendência com o incremento dos anos de escolaridade. Tal comportamento pode ser explicado devido ao fato de que indivíduos com mais anos de escolaridade possuam cargos com maior remuneração o que, consequentemente, leva a melhor condição financeira para a pratica de $\mathrm{AF}^{22}$. 
Indivíduos que afirmaram não fumar atualmente apresentaram maior chance de serem ativos no lazer em relação a seus pares. Indivíduos que fazem uso de tabaco apresentam uma resistência aeróbia reduzida em virtude da sobrecarga causada pelo cigarro no sistema cardiorrespiratório, o que pode influenciar de forma direta no abandono da prática de $\mathrm{AF}^{23}$. Além disso, esta associação pode ser afetada por viés de causalidade reversa.

Ainda no domínio do lazer foi possível observar que aqueles indivíduos categorizados como eutróficos apresentaram um comportamento mais ativo quando comparados as demais categorias analisadas. Estes resultados são corroborados pelos estudos de Olbrich et al. ${ }^{24} \mathrm{e}$ Conceição et al. ${ }^{25}$, os quais verificaram uma relação entre sobrepeso e obesidade com baixos níveis de AF em servidores federais. Essa diferença no nível de AF entre as categorias de IMC pode ser explicada devido aos motivos de adesão e desistência da prática citados por Weinberg \& Gould ${ }^{26}$. Os autores supracitados afirmam que entre os fatores internos e externos que influenciam no processo de realização de $\mathrm{AF}$, destaca-se a satisfação como um facilitador para a prática, onde o indivíduo realiza a atividade de forma prazerosa, acontecendo de forma mais natural entre os sujeitos com um IMC relativo a normalidade.Para os indivíduos com sobrepeso e obesidade, a AF representaria um esforço em busca da perda de peso ou melhora em saúde, e dessa forma deixaria de ser algo natural ou prazeroso, reduzindo a motivação para a manutenção ou início da prática.

Em relação ao domínio de deslocamento, o presente estudo observou que $26,2 \%$ dos técnicos foram ativos nesse domínio, valor maior que o encontrado pelo VIGITEL $^{9}$ para a população do estado do Rio Grande do Sul (12,8\%). Estudo de Hardman et al. ${ }^{27}$ identificou que $15,8 \%$ dos trabalhadores da indústria do estado de Pernambuco são fisicamente inativos nos deslocamentos para o trabalho. O fato do município de Pelotas apresentar uma geografia plana e a estrutura física da universidade ser multicêntrica pode ajudar nos deslocamentos ativos. Em relação à situação conjugal, técnicos solteiros foram mais ativos que seus pares, indo ao encontro dos achados de Polisseni \& Ribeiro ${ }^{18}$, em trabalhadores de uma universidade de Minas Gerais. De acordo com o estudo de Salles-Costa et al..$^{28}$ com 4.030 funcionários de uma universidade do Estado do Rio de Janeiro, indivíduos solteiros são mais ativos fisicamente, tanto mulheres quanto homens, por terem maior renda e mais tempo disponível para a prática de AF. Nesse sentido, mulheres solteiras têm menos res- ponsabilidades domésticas e familiares. Contudo, para os autores não há um consenso entre a relação prática de AF e situação conjugal, já que outros estudos afirmam que mulheres casadas ou com filhos apresentam maior percepção da importância da prática de AF para à saúde, indicando a necessidade de mais estudos sobre essa relação, principalmente considerando as diferenças entre os gêneros.

Técnico-administrativos do sexo masculino apresentaram mais chances de serem ativos no deslocamento, confirmando os achados de Madeira et al. ${ }^{29}$, os quais analisaram AF de deslocamento em estudo de base populacional com adultos e idosos de 23 estados do Brasil. Além disso, no presente estudo, com o aumento da idade aumentaram-se as chances de serem ativos fisicamente, o que contraria o estudo supracitado, o qual encontrou risco para inatividade física de deslocamento em sujeitos com mais idade. Diferentemente do estudo de Madeira et al. ${ }^{29}$, a faixa etária estudada no presente estudo possuía idade inferior, se encontrava atuante no mercado de trabalho e, mesmo realizando atividades não docentes, convivem em um meio acadêmico permeado sobre informações atualizadas do processo saúde-doença.

Uma das maneiras de tentar explicar os determinantes da prática de atividade física é através do modelo ecológico, que coloca a atividade física sob influência de atributos fisiológicos, psicológicos, sociais e ambientais. Entre os fatores ambientais está o ambiente construído (ciclovias, conectividade das ruas que facilite o acesso, padrão de calçadas e ruas, iluminação e segurança) o qual é importante fator de promoção da prática de atividade física principalmente no domínio do deslocamento. Nesse sentido, McCormack \& Shiell ${ }^{30}$, em estudo de revisão sistemática, demonstraram associação positiva entre ambiente construído e prática de atividade física no deslocamento. Apesar desta constatação, os autores salientam a necessidade de mais estudos com esta população e objetivando identificar determinantes para a prática de AF no domínio de deslocamento.

Algumas das limitações do presente estudo devem ser descritas. O mesmo foi realizado utilizando-se de um delineamento transversal, o qual impossibilita o entendimento de possíveis relações causais entre a atividade física e exposições avaliadas. Apesar dessa limitação, pontos fortes devem ser enfatizados. A utilização de um questionário com instrumentos validados e pré-testados, a uma cuidadosa logística de campo, a amostra representativa dos técnicos são pontos a serem destacados na pesquisa. Além disso, a população ava- 
liada ainda é pouco estudada na literatura, mostrando a importância dos dados obtidos no estudo.

Em resumo, a AF total foi maior entre técnico-administrativos do sexo masculino e de maior escolaridade. Em relação á pratica de lazer, indivíduos do sexo masculino, não fumantes, com maior nível de escolaridade e menor IMC foram mais ativos. No domínio de deslocamento, técnicos do sexo masculino, solteiros e com maior idade foram mais ativos nesse domínio. Com base nestes achados, intervenções em atividade física dentro e fora do ambiente de trabalho devem ser promovidas a fim de melhorar a saúde, qualidade de vida e, consequentemente, no desempenho de trabalho desses trabalhadores.

\section{Conflito de interesse}

Os autores declaram não haver conflito de interesse.

\section{Contribuição dos autores}

Santos TS, participou da interpretação dos dados, revisão crítica e aprovação da versão final. Lopes SV, participou da concepção e desenho do estudo, coleta, revisão crítica e aprovação da versão final. Caputo EL, participou da interpretação dos dados, revisão crítica e aprovação da versão final. Jerônimo JS participou da interpretação dos dados, revisão crítica e aprovação da versão final. Silva MC, participou da concepção e desenho do estudo, coleta e interpretação dos dados, revisão crítica e aprovação da versão final.

\section{Referências}

1. Gabriel KKP, Morrow Junior JRM, Woolsey AT. Framework for physical activity as a complex and multidimensional behavior. J Phys Act Health. 2012;9(1):11-18.

2. Lee IM, Shiroma EJ,Lobelo F, Puska P, Blair SN, Katzmarzyk PT. Lancet Physical activity series working group. Effect of physical inactivity on major non-communicable diseases worldwide: an analysis of burden of disease and life expectancy. Lancet. 2012;380:219-29.

3. Sallis JF, Bull F, Guthold R, Heath GW, Inoue S, Kelly P, et al. Lancet Physical activity series working group. Progress in physical activity over the Olympic quadrennium. Lancet. 2016;388:1325-36.

4. Papini CB, Nakamura PM, Martins CDO, Kokubun E. Severidades Ocupacionais associadas à inatividade física no lazer em trabalhadores. Motriz. 2010;16(3):701-07.

5. Barros MV, Nahas MV. Comportamentos de risco, auto-avaliação do nível de saúde e percepção de estresse entre trabalhadores da indústria. Rev Saúde Pública. 2001;35(6):554-63.

6. Ogata A, Bramante A, Costa L. Profissionais saudáveis, empresas produtivas: como promover um estilo de vida saudável no ambiente de trabalho e criar oportunidades para trabalhadores e empresas. Rio de Janeiro: Elsevier, 2012.

7. Nahas MV, Barros MVG, Oliveira ESA, Simm EE, Matos GAG. Lazer ativo: um programa de promoção de estilos de vida ativos e saudáveis para o trabalhador da indústria. Rev Bras Ativ Fís Saúde. 2010;15(4): 260-64.
8. Silva SG, Silva MC, Nahas, MV, Viana, SL. Fatores associados à inatividade física no lazer e principais barreiras na percepção de trabalhadores da indústria do Sul do Brasil. Cad de Saúde Pública. 2011;27(2):249-59.

9. Brasil. Ministério da Saúde. Vigitel Brasil 2015 Saúde Suplementar: vigilância de fatores de risco e proteção para doenças crônicas por inquérito telefônico [recurso eletrônico]/Ministério da Saúde, Agência Nacional de Saúde Suplementar. - Brasília: Ministério da Saúde. 2017.170 p.: il.

10. Höfelmann DA, Blank N. Auto-avaliação de saúde entre trabalhadores de uma indústria no sul do Brasil. Rev Saúde Pública. 2007;41(4):777-87.

11. Fonseca AS, Blank VLG, Barros MVG, Nahas MV.Percepção de saúde e fatores associados em industriários de Santa Catarina, Brasil. Cad de Saúde Pública. 2008;24(3):567-76.

12. Vandenbroucke JP, Von Elm E, Altman DG, Gøtzsche PC, Mulrow CD, Pocock SJ, et al. Strengthening the Reporting of Observational Studies in Epidemiology (STROBE): explanation and elaboration. PLoS Med. 2007;4(10):e297.

13. WHO. Obesity: Preventing and managing the global epidemic - Report of a WHO consultation on obesity. Geneva, 1998.

14. Ewing JA. Detecting alcoholism: The CAGE questionnaire. JAMA. 1984;252(14):1905-07.

15. Matsudo S, Araujo T, Matsudo V, Andrade D, Andrade E, Oliveira LC, Braggion G. Questionário internacional de atividade física (IPAQ): Estudo de validade e reprodutibilidade no Brasil. Rev. Bras. Ativ. Fis. Saúde. 2001; 6(2):5-18.

16. Haskell WL, Haskell WL, Lee IM, Pate RR, Powell KE, Blair SN, et al. Physical activity and public health: updated recommendation for adults from the American College of Sports Medicine and the American Heart Association. Med Sci Sports Exerc. 2007;39(8):1423-34.

17. Rocha SV,Pie ACS, Cardoso JP, Amorim CR, Carneiro LRV, Vilela ABA. Nível de atividade física entre funcionários de uma instituição de nível superior da Bahia. ACTA Brasileira do Movimento Humano. 2010; 1(3):16-29.

18. Polisseni MLC, Ribeiro LC. Exercício Físico como fator de proteção para a saúde em servidores públicos. Rev Bras Med Esporte. 2014;20(5):340-44.

19. Estilo de vida e hábitos de lazer dos trabalhadores das indústrias brasileiras: relatório geral / Markus V. Nahas ... [et al.]. - Brasília: SESI/DN, 2009.p. 163.

20. Florindo AA, Hallal, PC, Moura EC, Malta DC. Prática de atividades físicas e fatores associados em adultos, Brasil, 2006. Rev Saúde Pública. 2009;43(2):65-73.

21. Del Duca GF, Oliveira ES, Sousa TF, Silva KS, Nahas MV. Inatividade Física no lazer em trabalhadores da indústria do Rio Grande do Sul, Brasil. Motriz. 2011;17(1):180-88.

22. Sousa CA, Cesar CLG, Barros MBA, Carandina L, Goldbaum M, Marchioni DML, et al. Prevalência de atividade física no lazer e fatores associados: estudo de base populacional em São Paulo, Brasil, 2008-2009. Cad. Saúde Pública. 2013;29(2):270-82.

23. Nasser RL, Branco JC, Lara DR, Del Vecchio FB, Wiener C, Mesquita P, et al. Atividade Física de lazer e uso de substancias lícitas em uma amostra populacional de adultos jovens. Ciênc Saúde Coletiva. 2016;21(1):63-70.

24. Olbrich SRLR, Nitsche MJT, Mori NLR, Neto JO. Sedentarismo: prevalência e associação de fatores de risco cardiovascular. Rev Ciência em Extensão. 2009;5(2):30-41.

25. Conceição TVD, Gomes FA, Tauil PL, Rosa, TT. Valores de pressão arterial e suas associações com fatores de risco cardiovasculares em servidores da Universidade de Brasília. Arq Bras Cardiol. 2006;86(1):26-31.

26. Weinberg RS, Gould D. Fundamentos da psicologia do esporte e do exercício. 2 ed. Porto Alegre: Artmed, 2001. 
27. Hardman CM, Barros SSH, Oliveira ESA, Nahas MV, Barros MVG. Inatividade nos deslocamentos para o trabalho e fatores associados em industriários. Saúde Soc. 2013;22(3):760-72.

28. Salles-Costa R, Werneck GL, Lopes CS, Faerstein E. Associação entre fatores sócio-demográficos e prática de atividade física de lazer no Estudo Pró-Saúde. Cad. Saúde Pública. 2003;19(4):1095-105.

29. Madeira MC, Siqueira FCV, Facchini LA, Silveira DS, Tomasi E, Thumé E, et al. Atividade física no deslocamento em adultos e idosos do Brasil: prevalências e fatores associados. Cad. Saúde Pública. 2013;29(1):165-74.
30. McCormack G, Shiell A. In search of causality: a systematic review of the relationship between the built environment and physical activity among adults. Int J Behav Nutr Phys Act. 2011;8(125):6-11.
Recebido: 21/06/2017. Aprovado: 27/02/2018.

\section{Como citar este artigo:}

Santos TS, Lopes VL, Caputo EL, Jerônimo JS, Silva MC. Atividade física e fatores associados em técnico-administrativos de uma universidade pública do sul do Brasil. Rev Bras Ati Fis Saúde. 2018,23:e0028. DOI: 10.12820/rbafs.23e0028 